\title{
TURBULENT THERMAL CONVECTION IN A SPHERE
}

\author{
I. Cupal 1 , P. Hejda1 , M. Reshetnyak ${ }^{2}$ \\ 1 Geophysical Institute, Acad. Sci, 14131 Prague, Czech Republic \\ ${ }^{2}$ Institute of the Physics of the Earth, Russian Acad. Sci, 123995 Moscow, Russia \\ ${ }^{3}$ Research Computing Center of Moscow State University, 119899, Moscow, Russia
}

Convection in a rotating spherical layer in the Boussinesq approximation is considered. A free rotating solid concentric inner sphere is included. The problem of developed turbulence is solved using the shell model approach. Two sets of equations are used. The Navier-Stokes equation and the thermal flux equation are solved on a large scale due to a coarse grid. The small-scale solution is described by the shell model, which generates another set of equations. This enables us to estimate the spectral energy flux on small scales. The turbulent coefficients, depending on the radial direction, are then calculated and used in the large-scale solution. The behavior of other characteristics (spectra, helicity) is also studied in time and space. The stabilized solution of the largescale convection is obtained for the Rayleigh number $\mathrm{Ra}=10^{14}$ and the Ekman number $\mathrm{E}=10^{-6}$ based on the molecular values of viscosity and thermal diffusivity. The results correspond to the Reynolds number $\operatorname{Re} \sim 10^{9}$.

1. Introduction. The turbulent phenomena, appearing on small scales, complicate the computer simulation of convection in planetary interiors. Astrophysical and geophysical numerical models usually use a finite number, $N$, of the space grid points or of the spectral functions. $N$ cannot be too large as the computer process then becomes too long and results cannot practically be achieved. The control parameter for flows is the Reynolds number $\operatorname{Re}=V L / \nu_{\mathrm{M}}$, where $V$, $L$ are the characteristic velocity and length, respectively, and $\nu_{\mathrm{M}}$ is the molecular kinematic viscosity. Geodynamo models considering flows with $\operatorname{Re}=10^{9}[1]$ would required a grid with $N \approx \operatorname{Re}^{9 / 4} \approx 10^{20}$ nodes (e.g., [2]) for a small-scale turbulence to be resolved. Small-scale subgrid turbulent phenomena cannot be resolved by a coarse grid using ordinary computers. Of course, this does not mean that very interesting results cannot be obtained on the macro-scale, where a sensible large $N$ is used. There are many successful models of this kind. However, such models usually ignore the solution of the turbulent subgrid phenomena and only the parameters of the model are chosen basing on the theoretical consideration of subgrid turbulence. Nevertheless, the subgrid turbulence remains present during the numerical process, and the solution becomes unstable when it is not properly resolved on all scales. The numerical simulation becomes completely impossible and, therefore, the so-called hyperdiffusivity must be introduced (see, e.g., [3]) to avoid the influence of the small-scale turbulence, causing instabilities due to higher harmonics. This artificial hyperdiffusivity has to be introduced when models on the macro-scale are calculated.

Hyperdifusivity can be the solution of the problem for a spectral method. However, it is difficult to introduce something similar in the numerical process based on the space grid. In pure hydrodynamic problems solved on the macro-scale various semi-empirical models of turbulence were applied in the past. For example, the $K \varepsilon$-models (see, e.g., $[4,5])$ were often used in many scientific and technical applications. However, application to magnetohydrodynamics is not known and, 
moreover, models of this kind provide only averages over all wave numbers, characterizing the turbulence (e.g., viscosity, energy flux on a small scale), that is not convenient in magnetohydrodynamics. In this paper we attempt to solve only the hydrodynamic problem, however, still bearing in mind a later application in magnetohydrodynamics. Here we will use the shell model approach to the subgrid turbulence first introduced in [6], which was later generalized in [7] for complex variables and thus is now called the GOY model [8]. In principle, the shell model is a finite-dimensional model of turbulent motions based on the Fourier expansion, which gives a correct description of the spectral properties of the turbulent motions. Moreover, in $[9,10]$ the authors have developed the shell model approach for magnetohydrodynamics. Of course, the shell model requires appropriate computer facilities, but not a very powerful computer. Here we develop the model presented in $[11,12]$, where a conjunction of large and small scale models of thermal convection was done for the case of inhomogeneous turbulence. Due to different normalization of PDE, which provides a better numerical stability, we obtain a more critical regime of parameters. We also add the dependence of turbulence properties in the sphere on the $r$-direction.

2. Large-scale equations. Let the surface of a sphere of radius $r_{0}$ rotate with an angular velocity $\Omega$ about the $z$-axis. This sphere contains a concentric inner solid sphere of radius $r_{i}$, and the outer spherical layer $\left(r_{i}<r<r_{0}\right)$ is filled with an incompressible liquid $(\operatorname{div} \mathbf{v}=0)$. The inner sphere is allowed to rotate freely about the $z$-axis due to the viscous torque. The convection in the Boussinesq approximation in the outer sphere is described by the Navier-Stokes equation and by the heat flux equation. However, the usual form of these equations assumes that the diffusion coefficients do not depend on the space coordinates. We must assume the dependence of kinematic viscosity and thermal diffusivity on the space coordinates. Particularly, the kinematic viscosity and thermal diffusivity are composed of molecular and turbulent parts: $\nu_{\mathrm{M}}+\nu_{\mathrm{T}} \zeta(r), \kappa_{\mathrm{M}}+\kappa_{\mathrm{T}} \zeta(r)$. The molecular values are constant in space and time and the dimensionless function $\zeta(r)$ expresses the radial dependence of the turbulent values (see Section 3). Moreover, we also will assume that the turbulent Prandtl number $\operatorname{Pr}_{\mathrm{T}}=\nu_{\mathrm{T}} / \kappa_{\mathrm{T}}=1$. Introducing the small parameter $\beta=\nu_{\mathrm{M}} / \nu_{\mathrm{T}}$, we obtain $\kappa_{\mathrm{M}} / \kappa_{\mathrm{T}}=\beta \mathrm{Pr}^{-1}$, where $\operatorname{Pr}=\nu_{\mathrm{M}} / \kappa_{\mathrm{M}}$ is the molecular Prandtl number. Choosing $L=r_{0}$ as the unit of length, velocity $\mathbf{v}$, time $t$ and pressure $p$ can be measured in units of $\kappa_{\mathrm{T}} / L, L^{2} / \kappa_{\mathrm{T}}$ and $2 \Omega \rho \kappa_{\mathrm{T}}$, respectively. Denoting the dimensionless functions $\nu(r)=\beta+\zeta(r)$, $\kappa(r)=\operatorname{Pr}^{-1} \beta+\zeta(r)$, the dimensionless equations in the spherical coordinates $(r, \vartheta, \varphi)$ can be accepted in the form

$$
\begin{gathered}
\beta^{-1} \operatorname{Pr} \operatorname{Ro}\left[\frac{\partial \mathbf{v}}{\partial t}+(\mathbf{v} \operatorname{grad}) \mathbf{v}\right]=-\operatorname{grad} p+\mathbf{F}+\beta^{-1} \operatorname{Ediv}(\nu \overleftrightarrow{\mathbf{e}}) \\
\frac{\partial T}{\partial t}+\mathbf{v} \cdot \operatorname{grad}\left(T+T_{0}\right)=\operatorname{div}(\kappa \operatorname{grad} T)
\end{gathered}
$$

where force $\mathbf{F}$ is composed of the Coriolis and buoyancy forces

$$
\mathbf{F}=-\mathbf{1}_{z} \times \mathbf{v}+\beta P^{-1} \operatorname{Ra} \operatorname{Tr} \mathbf{1}_{r},
$$

$T_{0}$ denotes the prescribed temperature profile [13]:

$$
T_{0}=\frac{r_{i} / r-1}{1-r_{i}}
$$


and $\overleftrightarrow{\mathbf{e}}$ is the rate of the strain tensor. We note that

$$
\overleftrightarrow{\mathbf{e}}=e_{i k}=\frac{\partial v_{i}}{\partial x_{k}}+\frac{\partial v_{k}}{\partial x_{i}}, \quad \operatorname{div}(\nu \overleftrightarrow{\mathbf{e}})=\frac{\partial}{\partial x_{i}}\left(\nu e_{i k}\right)
$$

The dimensionless modified Rossby, Ekman and Rayleigh numbers based on the molecular values of kinematic viscosity and thermal difussivity appear in the equations

$$
\mathrm{Ro}=\frac{\kappa_{\mathrm{M}}}{2 \Omega L^{2}}, \quad \mathrm{E}=\frac{\nu_{\mathrm{M}}}{2 \Omega L^{2}}, \quad \mathrm{Ra}=\frac{\alpha g_{0} \Delta T L}{2 \Omega \kappa_{\mathrm{M}}},
$$

where $\alpha$ is the coefficient of thermal expansion, $g_{0}$ is the gravity acceleration at $r=$ $r_{0}$ and $\Delta T$ is the temperature drop through the outer liquid sphere. It should be mentioned that the Rayleigh number for non-rotating bodies is usually assumed in the form of $\widetilde{R a}=\alpha g_{0} \Delta T L^{3} / \kappa_{\mathrm{M}} \nu_{\mathrm{M}}$ and $\mathrm{Ra}=E \widetilde{R a}$. The Ekman number is small in the geophysical context, and one should keep in mind that $\mathrm{Ra} \ll \widetilde{\mathrm{Ra}}$. Similarly to "molecular" numbers, we can define the "turbulent" Rossby, Ro, Ekman, $\mathrm{E}_{\mathrm{T}}$ and Rayleigh, RaT numbers based on the turbulent values of kinematic viscosity and thermal diffusivity. The relations between the molecular and turbulent values are $\operatorname{Ro}=\beta \operatorname{Pr}^{-1} \operatorname{Ro}_{\mathrm{T}}, \mathrm{E}=\beta E_{\mathrm{T}}, \mathrm{Ra}=\beta^{-1} \operatorname{Pr} \mathrm{Ra}_{\mathrm{T}}$. The inner solid sphere is allowed to rotate freely about the $z$-axis due to the viscous torque. The dimensionless momentum equation for the angular velocity $\omega$ of the inner sphere $\left(0<r<r_{i}\right)$ has the form

$$
\operatorname{Pr} \operatorname{Ro} I \frac{\partial \omega}{\partial t}=r_{i} E \oint_{S}\left[\nu(r) e_{\varphi r}\right]_{r=r_{i}} \sin \vartheta \mathrm{d} S,
$$

where $I$ is the moment of inertia of the inner sphere, $S$ denotes its surface and the stress tensor component is

$$
e_{\varphi r}=\frac{\partial v_{\varphi}}{\partial r}+\frac{1}{r \sin \vartheta} \frac{\partial v_{r}}{\partial \varphi}-\frac{v_{\varphi}}{r} .
$$

Equations (1)-(4) are accompanied by the non-penetrating and no-slip boundary condition for velocity $\mathbf{v}$ and by the zero boundary condition for temperature perturbation $T$ at both $r=r_{i}$ and $r=r_{0}$.

Equations (1)-(4) describe properly the large scale convection provided that the grid mesh used for their solution is sufficiently fine. Problems arise when the solution changes to a scale smaller than the grid mesh $d$. Following Kolmogorov's assumptions, one has to provide a mesh, which would resolve scales $d_{\nu} \sim \operatorname{Re}^{-3 / 4}$ (e.g., [2]). The amplitude of the characteristic velocity $V$ of the convection is controlled by the amplitude of the buoyancy force. Namely, the viscous force is usually very small in volume and the Coriolis force influences the direction of velocity rather than its amplitude. Thus the estimate $\rho V^{2} \approx \rho \alpha g_{0} \Delta T L$ takes place using the dimensionalized Navier-Stokes equation. Hence, the Reynolds number relates to the modified Rayleigh number as $\mathrm{Re}=[\mathrm{Ra} /(\mathrm{EPr})]^{1 / 2}$, where $\operatorname{Pr}=\nu_{\mathrm{M}} / \kappa_{\mathrm{M}}$ is the (molecular) Prandtl number. Technical problems already arise if $\operatorname{Re}>10^{3}$ in the practical calculation of the $3 \mathrm{D}$ models. These problems can be solved with the approximate description of the turbulent effect for small-scale variables using the shell model of turbulence.

3. The shell model and its connection with the large-scale solution.

The idea of the shell model approach is to mimic the Navier-Stokes equation and the heat flux equation by a dynamical system with $n_{\max }$ variables $u_{1}, u_{2}, \ldots, u_{n_{\max }}$ and $\theta_{1}, \theta_{2}, \ldots, \theta_{n_{\max }}$, each representing the typical magnitude of velocity and temperature on a certain length scale. The Fourier space is divided into $n_{\max }$ shells 
and each shell $k_{n}$ consists of a set of wave vectors $\mathbf{k}$ so that $k_{0} 2^{n}<|\mathbf{k}|<k_{0} 2^{n+1}$, and thus each shell corresponds to an octave of the wave numbers. Variable $u_{n}$ is the velocity difference over length $\sim 1 / k_{n}$, so that there is one degree of freedom per shell. The coupling between shells is considered to preserve the main symmetries and properties of the Navier-Stokes equation. Particularly, the Coriolis force is neglected and also the gradient of pressure is not taken into account on small scales. Therefore, the inertia terms, the buoyancy force and viscous friction are only considered on small scales. Of course, the shell model is a simplified form of the turbulence description, nevertheless, it is a reasonable tool to study turbulence.

We will use the extension of the GOY model for the case of Boussinesq convection $[14,15]$ for the $3 \mathrm{D}$ case in real variables:

$$
\begin{aligned}
\beta^{-1} \operatorname{Pr} \operatorname{Ro} \frac{\mathrm{d} u_{n}}{\mathrm{~d} t}= & \beta^{-1} \operatorname{Pr} \operatorname{Ro} k_{n}\left(u_{n+1} u_{n+2}-\frac{1}{4} u_{n-1} u_{n+1}-\frac{1}{8} u_{n-2} u_{n-1}\right)+ \\
& +\beta \operatorname{Pr}^{-1} \operatorname{Ra} \theta_{n}-\operatorname{E} k_{n}^{2} u_{n}, \\
\frac{\mathrm{d} \theta_{n}}{\mathrm{~d} t}=k_{n} & \left(u_{n+1} \theta_{n+2}+u_{n-1} \theta_{n+1}-\frac{1}{2} u_{n-2} \theta_{n-1}+\theta_{n+1} u_{n+2}-\right. \\
& \left.-\frac{1}{2} \theta_{n-1} u_{n+1}-\frac{1}{4} \theta_{n-2} u_{n-1}\right)-\beta \operatorname{Pr}^{-1} k_{n}^{2} \theta_{n} .
\end{aligned}
$$

Even though the shell model equations (5) are an approximation of the original equations (1)-(2), they conserve the basic properties of these equations. The system of equations is characterized by three conservation laws in the force-free limit $(\mathrm{E}=0, \mathrm{Ra}=0)$. The first, $\mathcal{E}_{u}$, is the kinetic energy-like invariant and the second, $\mathcal{H}$, is the helicity-like invariant

$$
\mathcal{E}_{u}=\sum_{n}\left|u_{n}\right|^{2}, \quad \mathcal{H}=\sum_{n}(-1)^{n} k_{n}\left|u_{n}\right|^{2}, \quad \mathcal{E}_{\theta}=\sum_{n}\left|\theta_{n}\right|^{2}
$$

The latter condition is equivalent to the conservation of heat energy.

Equations (5) are integrated for $n=n_{\min }, \ldots, n_{\max }$. The lower limit $n_{\min }$ is determined by the grid density. Denoting $d$, the mean value of the distance between two neigboring grid points $k_{n_{\min }}=2 \pi / d$. The upper limit $n_{\text {max }}$ must make it possible to resolve the smallest scale appearing in the calculations, which is possible if the condition $k_{n_{\max }}>(\mathrm{Ra} / \mathrm{PrE})^{3 / 8}$ is satisfied.

The correct linkage (conjunction) between the large-scale solution of eqs. (1)(4) and the small-scale solution of eqs. (5) is a delicate point of this approach. Energy dissipation on the subgrid scale and a corresponding energy outflow from the large-scale solution must be evaluated. For this purpose the values of velocity and temperature (determined from the large-scale simulation) must be set at two adjacent points $u_{n_{\min }}, u_{n_{\min }+1}$ and $\theta_{n_{\min }}, \theta_{n_{\min }+1}$. These values reflect the current amplitude of the velocity and temperature oscillations on the typical large scale resolved by the grid. Denoting $\widehat{v}(k)$ and $\widehat{T}(k)$, the Fourier transform of the largescale velocity and temperature, respectively, the shell energy

$$
u_{n}^{2}=\int_{k_{n}}^{k_{n+1}}|\widehat{v}(k)|^{2} \mathrm{~d} k \approx k_{n}\left|\Delta v_{n}\right|^{2}, \quad \theta_{n}^{2}=\int_{k_{n}}^{k_{n+1}}|\widehat{T}(k)|^{2} \mathrm{~d} k \approx k_{n}\left|\Delta T_{n}\right|^{2}
$$

corresponds to the "energy" contained in the whole octave of the wave numbers. For convenience in the numerical process, $u_{n_{\min }}$ and $u_{n_{\min }+1}$ (as well as $\theta_{n_{\min }}$ and $\left.\theta_{n_{\min }+1}\right)$ are rather evaluated via the structural function of the second order, usually introduced in spectral methods. For the velocity that follows 
$S_{2}(h)=\left\langle[\mathbf{v}(x+h)-\mathbf{v}(x)]^{2}\right\rangle$ at point $x$, where $\langle\ldots\rangle$ denotes averaging over the whole calculated space, and similarly for the temperature. Assuming that the turbulence is spherically symmetric, the average is taken within a spherical layer of radius $r$. So, for each spherical layer we calculate its shell model. The other two directions are averaged. The assumption about the spherical symmetry of turbulence cannot be completely true, but meaningfully simplifies the computer processing. Nevertheless, for large Ra, not too fast rotation and for the given form of the temperature profile $T_{0}$ the assumption is usually true over the long time scales. By calculating the structural function $S_{2}$ for the scales corresponding to $k_{n_{\min }}, k_{n_{\text {min }}+1}$, we can find $u_{n_{\text {min }}}=\sqrt{S_{2}\left(h_{n_{\text {min }}}\right)}, u_{n_{\text {min }}+1}=\sqrt{S_{2}\left(h_{n_{\min }+1}\right)}$ and similarly for the temperature. These values are used in the shell model simulations (5). The influence of the large-scale solution on the small-scale subgrid turbulence is completed.

The reverse influence of the small-scale shell model on the large scales is also important. An effective (turbulent) viscosity $\nu_{\mathrm{T}}=\nu_{\mathrm{T}}(r)$ and a thermal diffusivity $\kappa_{\mathrm{T}}=\kappa_{\mathrm{T}}(r)$ are introduced. We will assume that under the regime of developed turbulence the mean amplitude $\nu_{\mathrm{T}} \approx \kappa_{\mathrm{T}}$ on each sphere of radius $r$ and hence $\nu_{\mathrm{T}}(r)=\kappa_{\mathrm{T}}(r)$. The turbulent Prandtl number $\operatorname{Pr}_{\mathrm{T}}=1$ on each sphere. The dissipation rate $\epsilon$ can be calculated as the total energy dissipated in the shell model per time unit (dimensionalized form)

$$
\epsilon=\nu_{\mathrm{M}} \sum_{n=n_{\min }+2}^{n_{\max }}\left(k_{n} u_{n}\right)^{2} .
$$

According to the Kolmogorov's assumption, the dissipation scale is $\sim\left[\left(\nu_{\mathrm{M}}\right)^{3} \epsilon^{-1}\right]^{1 / 4}$. Comparing the formulae with similar scales in the model of [16], an effective turbulent viscosity, which provides the same dissipation rate in the grid scale, is

$$
\nu_{\mathrm{T}}=0.1\left(d^{4} \epsilon\right)^{1 / 3}
$$

where $d$ is the coarse grid mean distance. Taking into account that velocity $u_{n}$, wave number $k_{n}$, and $d$ are measured in units of $\nu_{\mathrm{T}} / L^{2}, L^{-1}$ and $\mathrm{L}$, respectively, eq. (8) implies

$$
\zeta=0.1\left(\beta d^{4} \sum_{n=n_{\min }+2}^{n_{\max }} k_{n}^{2} u_{n}^{2}\right)^{1 / 3},
$$

where $d, k_{n}$ and $u_{n}$ are now considered to be dimensionless quantities. Thus equations (9) enable us to calculate (on each sphere of radius $r$ ) the dimensionless functions $\nu(r)$ and $\kappa(r)$ appearing in the large-scale equations (1) and (2).

4. Numerical methods and the results of simulation. The control volume method is used to solve eqs. (1)-(2) (for details see $[17,18]$ ). The number of nodes in the spherical staggered grid mesh $(r, \vartheta, \varphi)$ is $N=16$ in all three coordinates. However, the homogeneous spherical grid leads to inhomogeneous distances between the points and, therefore, the estimate of the minimal resolved scale $1 / k_{n_{\min }}$ is not trivial. We assume $d=1 / 16$. It should be noted that in the vicinity of the inner sphere surface and at the poles this scale is more than one order smaller and thus we choose $n_{\min }=4$ and $n_{\max }=24$ to provide resolution on the diffusion scales. The radius of the inner sphere is considered to be $r_{i}=0.35$ and $r_{0}=1$. We note that the turbulent time scale $t_{d}=(\mathrm{Ra} / \operatorname{Pr} \mathrm{E})^{-3 / 4}$ is smaller than the time step used in the large-scale simulation and, therefore, additional time of integration in the shell model is required. 


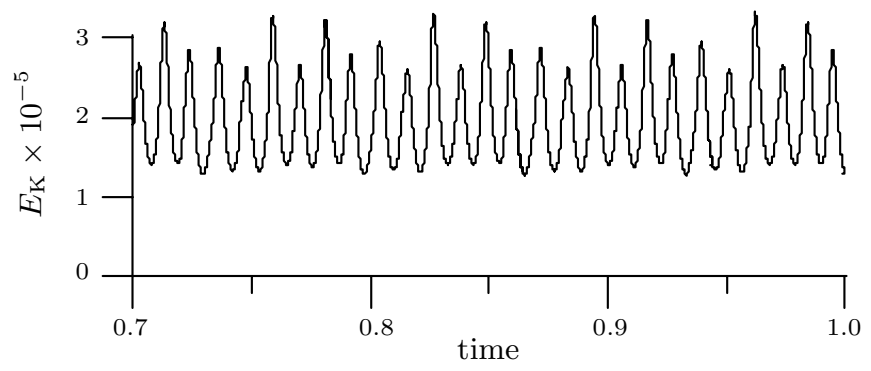

(a)

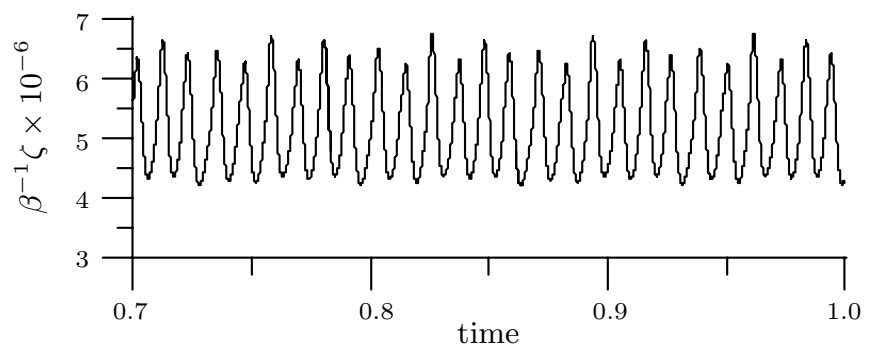

(b)

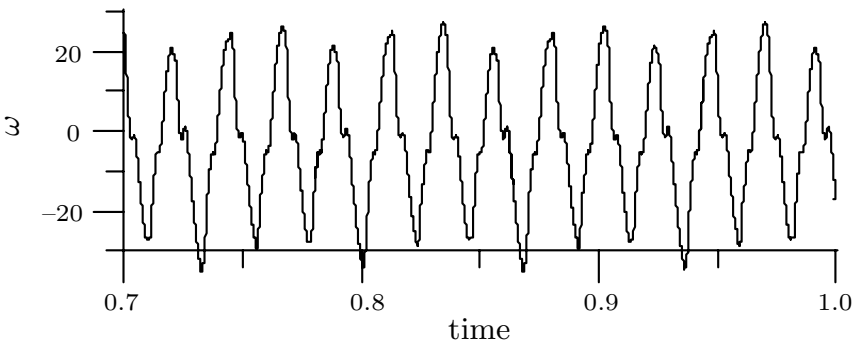

$(c)$

Fig. 1. (a) Evolution of the kinetic energy $E_{\mathrm{K}}$ of the large-scale motions, $(b)$ variations of the averaged over $r$ subgrid viscosity $\beta^{-1} \zeta$ and $(c)$ angular velocity $\omega$ of the inner shell plot with $\mathrm{Ra}=10^{9}$, Ro $=10^{-5}, \mathrm{E}=10^{-6}$.

The main part of the calculation is made on the computer cluster based on Pentium II computers with two processors. Standard Fortran-95 with MPI for parallelization is used. The algorithm of the calculations uses the main processor to calculate the large-scale equation and the results are the velocity and temperature for the minimum resolved grid scale. The obtained values $u_{n_{\min }}, u_{n_{\min }+1}$ $\left(\theta_{n_{\min }}, \theta_{n_{\min }+1}\right)$ are used in the shell model and distributed over the processors responsible for the turbulent simulation. At each node of the grid in the radial coordinate a different processor calculates the shell model. The obtained viscosity for each $r$-node is returned to the main processor and used in large-scale simulation. Therefore, the time needed to calculate the turbulence is of the same order as (or larger than) the time of the large-scale model calculation. All processors were used effectively all the time and the exchange of data between the computers was one-dimensional only $(\sim N)$ and not time consuming.

Below we demonstrate our calculations for $\beta=10^{-5}$, Ro $=10^{-5}, \mathrm{E}=10^{-6}$, $\mathrm{Ra}=10^{14}$. In our calculations we proceed with more than one diffusion time, but for convenience of presentation only some time sections of the simulations are presented. Fig. 1 illustrates the evolution of some averaged over the spherical volume parameters. Fig. $1 a$ corresponds to a large-scale kinetic energy $E_{\mathrm{K}}$. Note 
that the mean level of kinetic energy $2 \times 10^{5}$ corresponds to Re $\sim 10^{9}$ based on molecular viscosity. This should be considered as a very supercritical regime (at least for the given grid mesh). Turbulence with such Reynolds number could be resolved using direct numerical simulations (DNS) only with more than $5 \times 10^{6}$ nodes in one direction that is beyond the computing possibilities of contemporary computers. Fig. $1 b$ shows the variation of the subgrid turbulent viscosity $\beta^{-1} \zeta$. The mean level of this quantity is about $5 \times 10^{6}$ that corresponds to the turbulent (effective) modified Rayleigh, Ekman and Rossby numbers Ra $\sim 10^{10}, \mathrm{E}_{\mathrm{T}}=$ 5 and $\mathrm{Ro}_{\mathrm{T}}=50$, respectively. Note that the molecular Prandtl number was chosen to be $\operatorname{Pr}=0.1$. Fig. $1 c$ corresponds to the evolution of the inner sphere's angular velocity $\omega$. As far as our regime corresponds to the case of a very slow rotation when turbulence is taken into account $\left(\mathrm{E}_{\mathrm{T}}=5\right)$, we do not find any preference in the direction of $\omega$. We remind the reader that for the cases when $\mathrm{E} \ll 1$ and the angular drift of the vertical Busse's columns starts [13], the mean level of $\omega$ can differ from zero. These speculations are in agreement with the spatial maps of the temperature and velocity fields illustrated in Fig. 2 (for $t=1)$. These plots demonstrate a very regular behavior of the quantities without small-scale perturbations. We also observe the absence of an Ekman layer (see, in particular, $v_{\varphi}$ ). If the calculations have been made with the corresponding values of molecular kinematic viscosity and thermal diffusivity, highly irregular structures in the fields would be observed (of course, if the numerical scheme is still stable).

Fig. 3 shows the averaged over the shell volume spectral characteristics for both grid and subgrid scales for the velocity and temperature fields. The large (grid) scale is described by a second-order structure function $S_{2}$ (black circles). The small (subgrid) scale is characterized by a mean shell energy $\sim\left\langle u_{n}^{2}\right\rangle,\left\langle\theta_{n}^{2}\right\rangle$ (black squares). The conjunction between the grid and subgrid scales occurs at two points with $n_{\min }=4$ and $n_{\min }+1=5$. The tails of the structure function $(n=5 \div 8)$ correspond to polar regions, where the spherical grid has a better resolution. Although we did not force the fields to have the same values for $n>5$ for the grid and shell models, their similarity for the both models is obvious. Considering the shell model spectra, we define the end of the inertial interval of
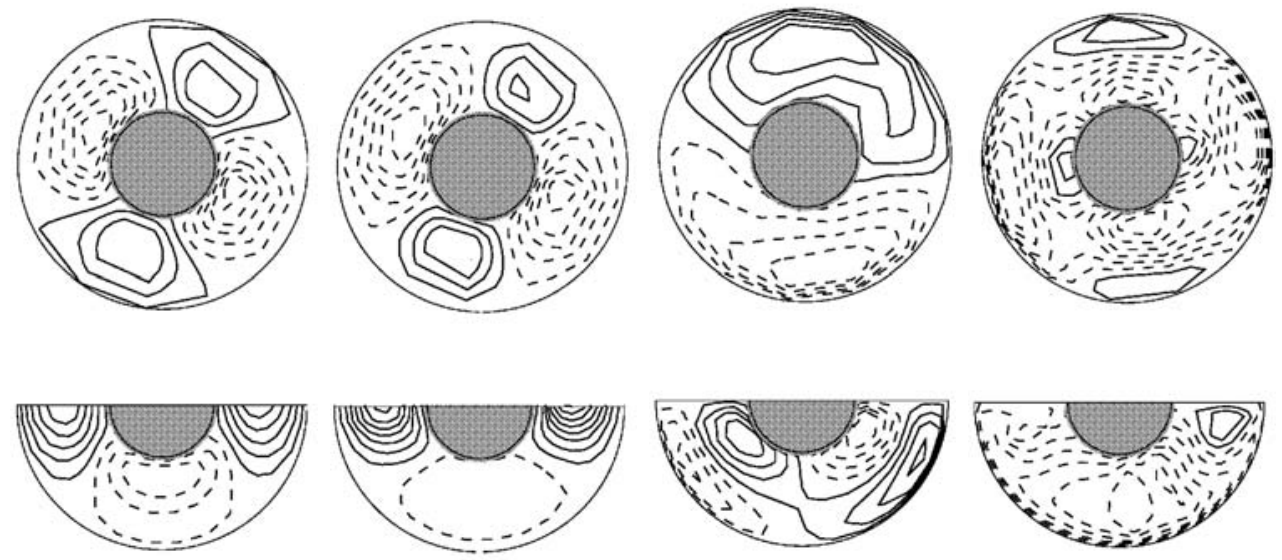

Fig. 2. From left to right: the snapshots of the temperature field $T$ and velocity field components $\left(v_{r}, v_{\vartheta}, v_{\varphi}\right)$ for the equatorial sections (top row): $(-0.28,0.14),(-138,94)$, $(-356,308),(-203,87)$, and meridional sections for axi-symmetrical parts of the fields (bottom row): $(-0.1,0.1),(-35,228),(-109,104),(-101,50)$. Numbers in round brackets indicate ranges. The time moment corresponds to $t=1$. 

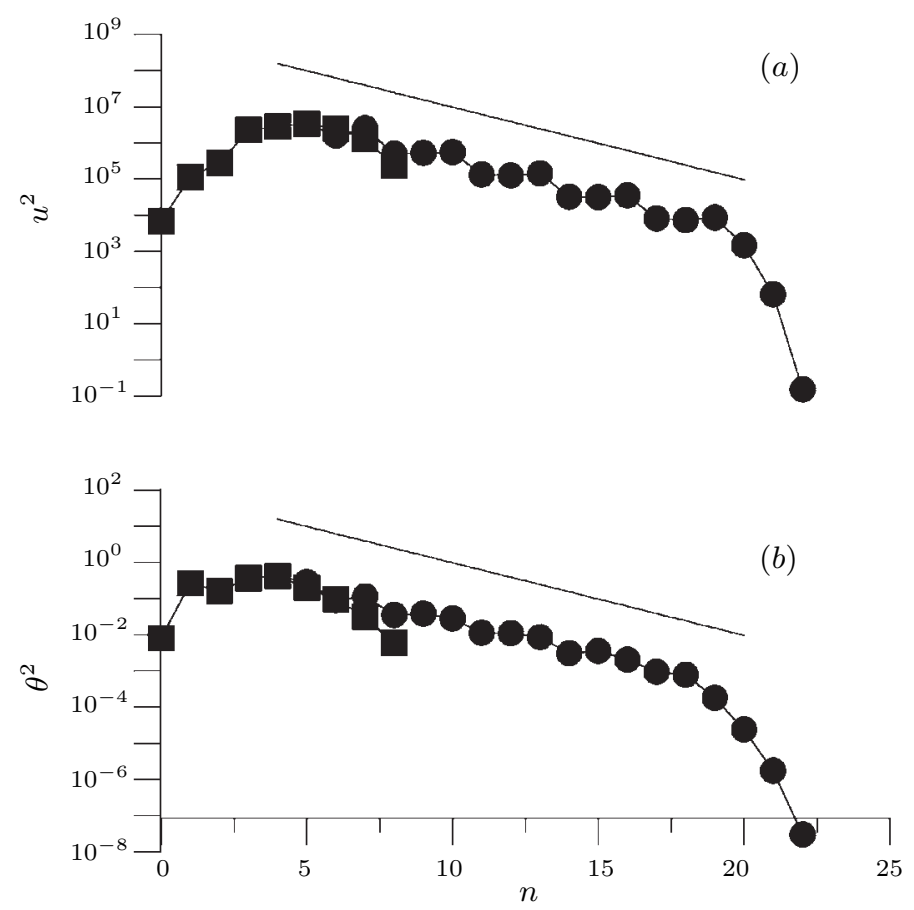

Fig. 3. Structure function $S_{2}$ (squares) and shell spectrum (circles) for the turbulent velocity $(a)$ and temperature $(b)$. The straight line corresponds to the Kolmogorov's spectrum.

the both spectra for $u$ and $\theta$ to be the same at $n=19$. The slope of the spectra is very close to the Kolmogorov's one " $-5 / 3$ ". Straight lines in the both plots show these spectra.

As already mentioned, for every radial layer we used its own shell model in our simulations. Fig. 4 shows turbulent viscosity $\zeta$ as a function of radial distance $r$. The values are averaged over $\vartheta$ and $\varphi$. The plot shows that the diffusion maximum repeats the energy distribution over the $r$-direction. As follows from the plots of space distributions of the velocity and temperature fields in Fig. 2, main energy corresponds to position $r=0.7 \div 0.8$ is in agreement with the maximum of diffusion $(\zeta)$ generation in Fig. 4. Other data can be found in the 2D-picture of spatial-

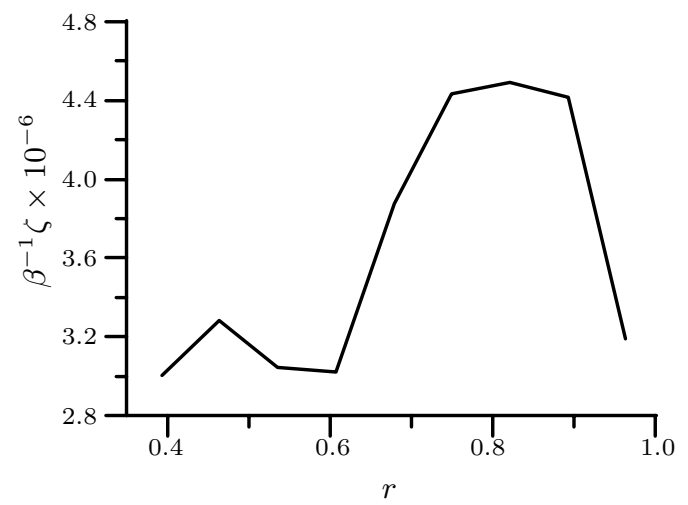

Fig. 4. The spatial distribution of turbulent diffusion in the spherical layer. 

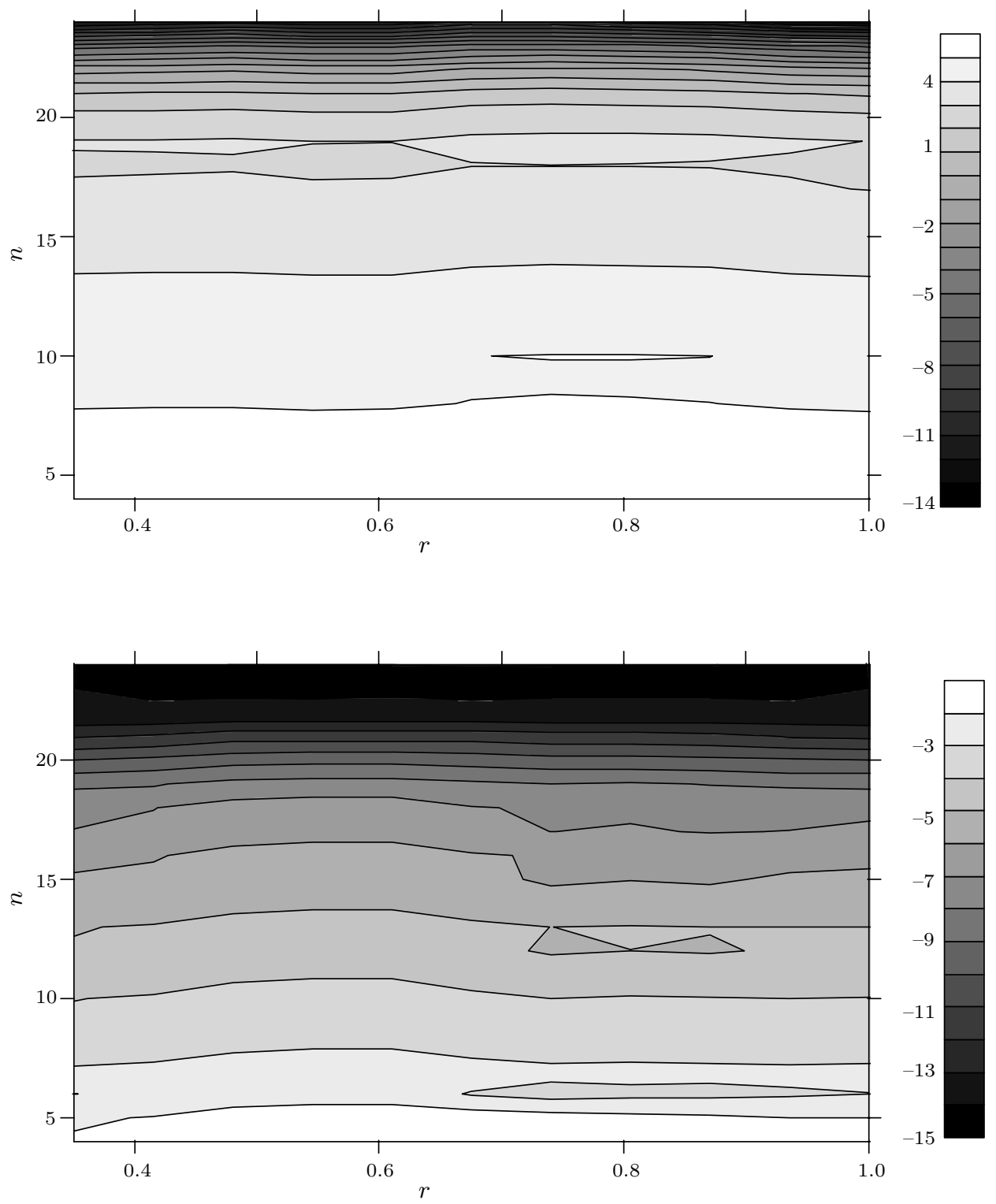

Fig. 5. Dependence of the turbulent velocity (upper) and temperature (lower) spectra $\left(\log _{10} u_{n}^{2}, \log _{10} \theta_{n}^{2}\right)$ on the radial direction for different $n$.

frequency distribution of small-scale velocity and temperature (Fig. 5).

5. Conclusions. The approach developed in this paper allows the spectral properties to be resolved in the whole range of subgrid scales for given Rayleigh and Rossby numbers. It is believed that this approach, after a moderate increase of the number of shells, can be applied to the range of Rayleigh and Rossby numbers, which are of common interest to geophysics and astrophysics. Note that our results correspond to $\operatorname{Re} \sim 10^{9}$, which already has the same magnitude as is expected in the Earth. The presented model of large-scale convection is a promissing way for describing the subgrid turbulence due to the solution of the shell model (a 
grid-shell approach). In contrast to the usual semi-empirical models, which provide only large-scales quantities, this approach provides much more information about the small-scale phenomena.

The model of convection should be considered as the first step on the way to the hydromagnetic dynamo model. The control volume method has already been successfully used for simulating the large-scale hydromagnetic dynamo [18], and, therefore, we plan to include also the Lorentz force in the shell model. The problem has already been considered in the shell-model equations in $[9,10,19]$. We assume the Coriolis force to be neglected in the shell model. This is a reasonable simplification as this term does not put any energy into the system and takes part only in the redistribution of kinetic energy. Of course, the full model should include the effects of anisotropy discussed in detail in [20] and often considered in astrophysical applications [21]. We predict two possibilities how to do it. The most systematic way is to produce the shell model equations for each vector component of the original PDE. Then, in principal, all required properties of the equations could be provided. The other way is to use some information about integral spectra and to consider only one "integral" shell equation for all three components. Following the model developed in [22], one can introduce a dependence of the spectra on rotation and then only some moderate modification of our present model is required.

We also realize that the structure function is not the only tool, which can be used for matching large- and small-scale solutions, and that some more comparison of our results with the analytical and empirical predictions is required. Our main goal here was to introduce a general idea of such matching of the two models and we are continuing with our studies in this field.

Acknowledgements. The authors are grateful to P. Frick and D. Sokoloff for numerous discussions of the problem. This work was supported by the Russian Foundation of Basic Research (Grant 03-05-64074) and by the Grant Agency of the Academy of Sciences of the Czech Republic (Grant A3012006).

\section{REFERENCES}

[1] D. Gubbins, P.H. Roberts. Magnetohydrodynamics of the Earth's core. In Geomagnetism (Ed. Jacobs, Academic Press), vol. 2, pp. 1-184.

[2] U. Frisch. Turbulence: the Legacy of A.N. Kolmogorov (Cambridge University Press, Cambridge, 1995).

[3] K. Zhang, C.A. Jones. The effect of hyperviscosity on geodynamo models. Geophys. Res. Lett., vol. 24 (1997), pp. 2869-2872.

[4] C.A. Fletcher. Computational Techniques for Fluid Dynamics (SpringerVerlag, NY, 1988).

[5] W. Kollman. Prediction Methods for Turbulent Flows (Hemisphere Publishing Corporation, 1980).

[6] E.B. Gledzer. System of hydrodynamic type admitting two quadratic integrals of motion. Sov. Phys. Dokl. vol. 18 (1973), no. 4, pp. 216-225.

[7] K. Ohkitani, M. Yamada. Temporal intermittency in the energy cascade process and local Lyapunov analysis in fully developed model turbulence. Prog. Theor. Phys., vol. 81 (1989), no. 2, pp. 329-334. 
[8] T. Bohr, M. Jensen, G. Paladin, A. Vulpiani Dynamical Systems Approach to Turbulence (Cambridge University Press, Cambridge, 1998).

[9] P. Frick, D. Sokoloff. Cascade and dynamo action in a shell model of magnetohydrodynamic turbulence. Phys. Rev. E, vol. 57 (1988), pp. 41554164 .

[10] P. Frick, M. Reshetnyak, D. Sokoloff. Cascade models of turbulence for the Earth's liquid core. Earth Sciences. vol. 387 (2002), no. 8, pp. 988991.

[11] P. Frick, M. Reshetnyak, D. Sokoloff. Combined grid-shell approach for convection in a rotating spherical layer. Europhys. Lett. vol. 59 (2002), pp. 212-217.

[12] M. Reshetnyak, P. Frick, D. Sokoloff. Combined grid-shell approach for convection problem in a spherical layer. In Advances in Turbulence IX, Proceedings of the Ninth European Turbulence Conference, (Eds. I.P. Castro, P.E. Hancock \& T.G. Thomas, Barcelona, 2002), pp. 283-286.

[13] A. Tilgner, F.H. Busse. Finite amplitude in rotating spherical fluid shells. J. Fliud. Mech., vol. 332 (1997), pp. 359-376.

[14] J. Mingshun, L. ShidA. Scaling behavior of velocity and temperature in a shell model for termal convective turbulence. Physycal Review E, vol. 56 (1997), no. 1.

[15] S. A. Lozhkin, P. G. Frick. Inertial Obukhov-Bolgiano interval in shell models of convective turbulence. Fluid Dynamics, vol. 33 (1998), no. 6, pp. $125-140$.

[16] J. Smagorinsky. General circulation experiments with the primitive equations: I. The basic experiment. Monthly Weather Review, vol. 91 (1963), pp.99-164.

[17] S.V. Patankar. Numerical Heat Transfer And Fluid Flow (Taylor \& Francis, 1980).

[18] P. Hejda, M. Reshetnyak. Control volume method for the dynamo problem in the sphere with the free rotating inner core. Studia geoph. et geod., vol. 47 (2003), pp. 147-159

[19] P. Frick, B. Dubrulle, A. Babiano. Scaling properties of a class of shell models. Phys. Rev. E, vol. 51 (1995), pp. 5582-5593.

[20] S.I. Braginsky, V.P. Meytuis 1990 Local turbulence in the Earth's core. Geophys. Astrophys. Fluid Dynamics, vol. 55 (1990), pp. 71-87.

[21] G. RÜDiger, L.L. Kichatinov Alpha-effect and alpha-quenching. Astronomy and Astrophysics, vol. 269 (1993), pp.581-588.

[22] Y. Zhou. A phenomenological treatment of rotating turbulence. Phys. Fluids, vol. 7, no. 8, (1995), pp. 2092-2094.

Received 16.05.2003 